\title{
Numerical upscaling of frequency-dependent P- and S-wave moduli in fractured porous media
}

\author{
Eva Caspari ${ }^{1 *}$, Marco Milani ${ }^{1}$, J. Germán Rubino ${ }^{2}$, Tobias M. Müller ${ }^{3}$, \\ Beatriz Quintal $^{1}$ and Klaus Holliger ${ }^{1}$ \\ ${ }^{1}$ Applied and Environmental Geophysics Group, Institute of Earth Sciences, University of Lausanne, 1015 Lausanne, Switzerland, \\ ${ }^{2}$ Department of Earth Sciences, University of Western Ontario, London, ON N6A5B7, Canada, and ${ }^{3}$ CSIRO Energy, Perth, Australia
}

Received September 2015, revision accepted February 2016

\begin{abstract}
Relating seismic attributes to the characteristics of mesoscopic fractures is inherently challenging, yet these heterogeneities tend to dominate the mechanical and hydraulic properties of the medium. Analytical approaches linking the effects of material properties on seismic attributes, such as attenuation and velocity dispersion, tend to be limited to simple geometries, low fracture densities, and/or non-interacting fractures. Furthermore, the influence of fluid flow within interconnected fractures on P-wave and S-wave attenuation is difficult to accommodate in analytical models. One way to overcome these limitations is through numerical upscaling. In this paper, we apply a numerical upscaling approach based on the theory of quasi-static poroelasticity to fluid-saturated porous media containing randomly distributed horizontal and vertical fractures. The inferred frequency-dependent elastic moduli represent the effective behaviour of the underlying fractured medium if the considered sub-volume has at least the size of a representative elementary volume. We adapt a combined statistical and numerical approach originally proposed for elastic composites to explore wether the overall statistical properties of simple fracture networks can be captured by computationally feasible representative-elementary-volume sizes. Our results indicate that, for the considered scenarios, this is indeed possible and thus represent an important first step towards the estimation of frequency-dependent effective moduli of realistic fracture networks.
\end{abstract}

Key words: Rock physics, Equivalent media, Fracture.

\section{INTRODUCTION}

The hydraulic characterization of fractured rocks based on seismic data is widely regarded as something like an ultimate frontier in geophysics, yet it has a number of important applications, such as, for example, the sustainable use of groundwater, the optimized production of hydrocarbons and geothermal energy, and the safe storage of nuclear waste. While fractures tend to control the mechanical and hydraulic properties of the medium, they can in general not be resolved

*E-mail: eva.caspari@unil.ch directly, which makes it challenging to relate measured seismic attributes to the characteristics of the fractured rocks.

Numerous analytical effective medium theories were proposed to relate the effects of fractures to the overall elastic and hydraulic properties of the considered media (e.g., Kachanov 1992; Schoenberg and Sayers 1995; Sanchez-Vila, Guadagnini, and Carrera 2006). Recent studies have shown that attenuation and velocity dispersion of seismic waves are sensitive to the elastic and hydraulic properties and thus may provide critical insights into fracture characteristics (e.g., Chapman 2003; Brajanovski, Müller, and Gurevich 2006; Gurevich et al. 2009). However, analytical effective medium 
models tend to be inherently limited to simple geometries, low fracture densities, and/or non-interacting fractures, criteria which are generally not fulfilled by actual fracture networks.

One way to overcome the limitations of analytical approaches is through numerical upscaling procedures based on spatial averaging. These methods have been used for various rock physical properties, e.g., elastic, electrical, and hydraulic properties (e.g., Saenger, Krüger, and Shapiro 2006; OstojaStarzewski 1999, 2006; Willot and Jeulin 2011). Recently, the upscaling of the seismic characteristics of porous media, such as velocity dispersion and attenuation, based on the theory of poroelasticity has gained increasing acceptance and popularity (e.g., Masson and Pride 2007; Rubino, Ravazzoli, and Santos 2009; Wenzlau, Altmann, and Müller 2010; Quintal et al. 2011). In these numerical approaches, a homogeneous displacement or stress field is applied to the boundaries of a subvolume of a heterogeneous poroelastic medium. By spatial averaging of the resulting complex stress and strain fields, the phase velocities and the attenuation as functions of frequency can then be inferred. These upscaling procedures are restricted to a finite sub-volume of the considered medium. Correspondingly, the upscaled physical properties describe the effective behaviour of the underlying heterogeneous medium only if the probed sub-volume has at least the size of a socalled representative elementary volume (REV). The adequate definition of a REV for a given upscaling problem is therefore of critical importance.

Hill's (1965) classical work for elastic composites defines a REV as (i) a sub-volume for which the inferred average properties are independent of the applied boundary conditions and (ii) that is structurally representative of the entire medium. Jänicke, Quintal, and Steeb (2015) extended Hill's (1965) approach to derive a consistent upscaling procedure of a heterogeneous poroelastic medium to a viscoelastic medium. A main focus of their investigation was boundary condition effects on the fluid pressure, which can cause a shift in frequency of the attenuation maximum. Recently, Milani et al. (2016) explored the definition of a REV for periodically fractured porous media and analysed in detail the corresponding boundary condition effects on the stress and strain fields. They found that the REV size depends on the compressibility contrast between the fractures and their embedding background and on the fracture density.

In this paper, we extend the analysis of Milani et al. (2016) to porous media containing randomly distributed horizontal and vertical fractures. The question then arises if the considered sub-volumes represent the overall statistical properties of the underlying heterogeneous fractured medium. In the context of elastic composite media, Kanit et al. (2003) addressed this problem through a combined statistical and numerical approach based on the concept of the integral range. This method provides estimates of the REV size for a given relative error of the moduli and a given number of realizations of the considered random media by fitting a scaling law to simulations of several realisations and domain sizes. Here, we adapt this approach to porous media with randomly distributed fractures. This provides a critical step towards the estimation of frequency-dependent effective moduli of realistic fracture networks.

In the following, we first describe the properties of the considered fractured media and then proceed to present the numerical upscaling procedure, the associated characteristic length scales, and the statistical approach. Finally, we apply the combined statistical and numerical approach to $2 \mathrm{D}$ poroelastic media with randomly distributed fractures and assess the corresponding results.

\section{FRACTURED POROUS ROCKS MODELLED AS POROELASTIC MEDIA}

We consider 2D numerical models of a water-saturated tight sandstone containing randomly distributed horizontal and vertical fractures. This is equivalent to corresponding 3D models under plane strain conditions, for which the displacements out of the modelling plane are zero. For simplicity, we only consider uniform distributions and thus uncorrelated fracture centres. Four different scenarios are analysed (Fig. 1): uniform distributions of fractures of the same size for two fracture densities $\left(\rho_{\text {frac }}=0.16\right.$ and $\left.\rho_{\text {frac }}=0.32\right)$ and uniform distributions of fractures of variable length for parallel fractures and orthogonal fracture sets. The fracture density is calculated as $\rho_{\text {frac }}=\left(\sum_{N} 0.5 l_{\text {frac }}^{2}\right) / S$, where $N$ is the number of fractures, $l_{\text {frac }}$ is the fracture length, and $S$ is the domain size (Kachanov 1992). The fractures have a rectangular geometry with a constant aperture $(a=0.06 \mathrm{~cm})$, regardless of their lengths $\left(l_{\text {frac } 1}=2.4 \mathrm{~cm}, l_{\text {frac } 2}=4.8 \mathrm{~cm}, l_{\text {frac } 3}=9.6 \mathrm{~cm}\right)$.

The uniform distribution of fracture centres is created by drawing $n$ integers from a discrete uniform distribution in the interval from $[1, n x \times n y]$, where $n x$ and $n y$ are integers proportional to the side lengths of the sample. These random numbers are then mapped to $x$ - and $y$-coordinates and successively placed into the model area discarding overlapping fractures and replacing them by newly generated fracture centres. Although, in a strict sense, this violates the condition of uncorrelated fracture centres, the number of rejected fractures 
(a)

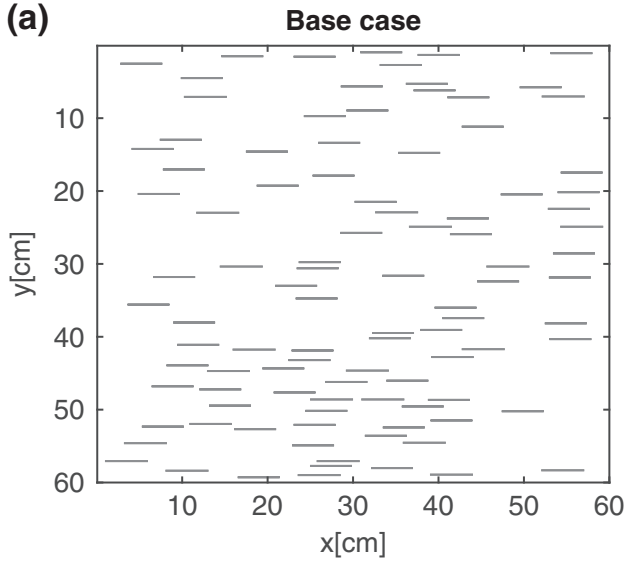

(c)

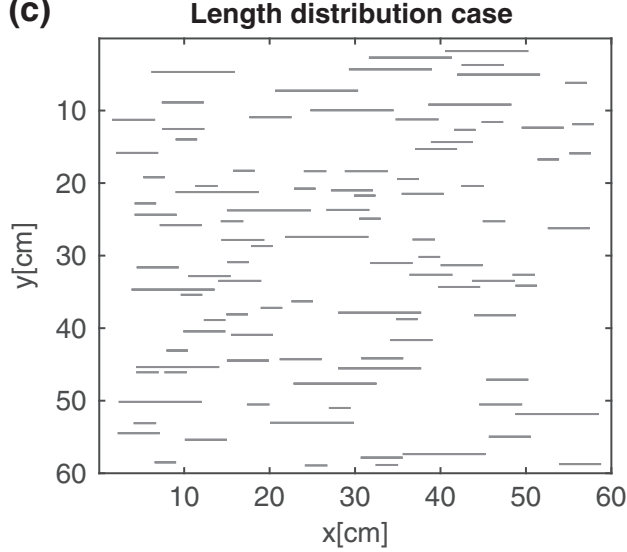

(b)

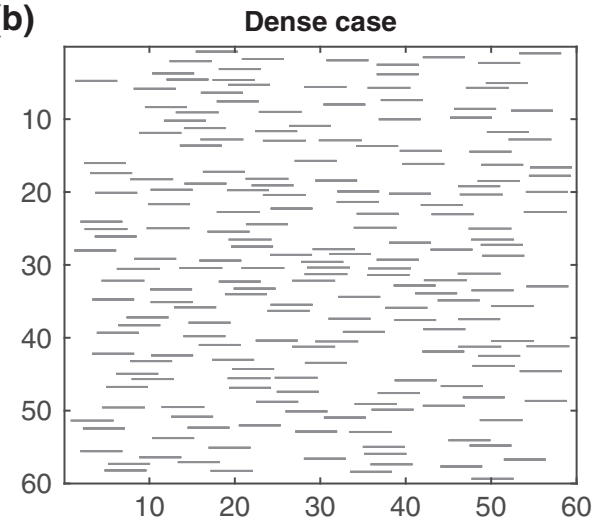

(d)

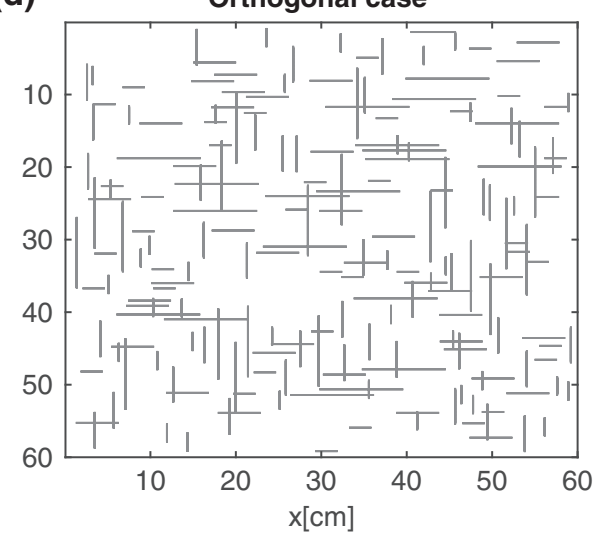

Figure 1 Examples of uniform fracture distributions considered in this study: fractures of the same size with a fracture densities of (a) $\rho_{\text {frac }}=0.16$ (base case) and (b) $\rho_{\text {frac }}=0.32$ (dense case), and fractures of variable length for (c) parallel fractures (length distribution case) and (d) orthogonal fracture sets (orthogonal case).

is generally small and can hence be assumed not to create a systematic error. This procedure is performed independently for horizontal and vertical fractures and thus we are not controlling the number of connected fractures in the orthogonal set.

The tight-sandstone and the embedded fractures are represented as a poroelastic continuum. The sandstone is characterized by a stiff porous matrix of low permeability and porosity. Traditionally in elastic media, fractures are described as discontinuities by their aperture and their normal and tangential compliances (Kachanov 1992; Schoenberg and Sayers 1995). In this study, we represent the fractures as strongly compliant poroelastic features, which in turn implies that pore-scale characteristics must enter the macroscopic poroelastic equations in an averaged form. Therefore, in addition to the compliances, estimates of the fracture porosity and permeability are needed. Given that fractures do not tend to be entirely void and that their walls do not correspond to smooth parallel planes, we choose a somewhat lower permeability than that given by the analytical solution of the Navier-Stokes equation for laminar flow between two smooth walls with a uniform aperture (Jaeger, Cook, and Zimmerman 2007). Moreover, we choose high compliance and very high porosity, which results in a large stiffness contrast with respect to the embedding sandstone matrix. The rock properties are summarized in Table 1.

\section{NUMERICAL UPSCALING IN POROELASTICITY}

The numerical upscaling procedure is based on Biot's (1941) quasi-static poroelastic equations, in which inertial coupling between the solid and fluid motion is neglected. The quasistatic approximation is applicable if the highest considered frequency is much smaller than Biot's critical frequency at which viscous and inertial forces equally act on the pore 
Table 1 Physical properties of the fractures, the embedding background, and the saturating pore fluid. Adopted from Rubino et al. (2013) and Milani et al. (2016)

\begin{tabular}{|c|c|c|c|c|}
\hline & Bulk modulus, $K$ & Shear modulus, $\mu$ & Porosity, $\phi$ & Pemeability, $\kappa$ \\
\hline Background & $26.0 \mathrm{GPa}$ & $31.0 \mathrm{GPa}$ & 0.10 & $1.0 \mathrm{mD}$ \\
\hline \multirow[t]{2}{*}{ Fracture } & $0.03 \mathrm{GPa}$ & $0.02 \mathrm{GPa}$ & 0.80 & $100 \mathrm{D}$ \\
\hline & Bulk modulus, $K$ & Density, $\rho$ & Viscosity, $\eta$ & \\
\hline Grain & $37 \mathrm{GPa}$ & $2650 \mathrm{~kg} / \mathrm{m}^{3}$ & & \\
\hline Water & $2.25 \mathrm{GPa}$ & $1000 \mathrm{~kg} / \mathrm{m}^{3}$ & $1.0 \cdot 10^{-3} \mathrm{~Pa} \cdot \mathrm{s}$ & \\
\hline
\end{tabular}

fluid (Mavko, Mukerji, and Dvorkin 2009). In the quasi-static regime, dissipation of wave energy can be attributed to the viscous coupling of the elastic solid and the fluid resulting in an out-of-phase relative fluid-solid motion. The associated slow P-wave behaves as a pressure diffusion wave.

\section{Quasi-static poroelasticity equations}

The system of two coupled equations in the frequency domain is given by the stress equilibrium and Darcy's law, i.e.,

$$
\nabla \cdot \sigma=0
$$

$-\nabla p_{f}=i \omega \frac{\eta}{\kappa} \mathbf{w}$

where $\sigma$ and $p_{f}$ are the total stress tensor and fluid pressure, respectively, and $\omega$ is the angular frequency. The relative fluid-solid displacement is given by $\mathbf{w}=\phi(\mathbf{U}-\mathbf{u})$, where $\phi$ is the porosity, and $\mathbf{U}$ and $\mathbf{u}$ are the macroscopic fluid and solid displacements, respectively. The ratio of the permeability $\kappa$ and the fluid shear viscosity $\eta$ denotes the hydraulic conductivity. The constitutive equations of the poroelastic medium are

$$
\begin{aligned}
\sigma & =[(H-2 G) \nabla \cdot \mathbf{u}+\alpha M \nabla \cdot \mathbf{w}] \mathbf{I}+G\left[\nabla \mathbf{u}+(\nabla \mathbf{u})^{T}\right], \\
-p_{f} & =\alpha M \nabla \cdot \mathbf{u}+M \nabla \cdot \mathbf{w},
\end{aligned}
$$

where I denotes the identity tensor, and $G$ is the shear-wave modulus of the porous material. The undrained low-frequency P-wave modulus is defined as $H=L+\alpha^{2} M$, with $L$ denoting the dry P-wave modulus, $\alpha=1-K_{d} / K_{s}$ the Biot-Willis coefficient, and $M=\left[(\alpha-\phi) / K_{s}+\phi / K_{f}\right]^{-1}$ the fluid storage coefficient. The parameters $K_{d}, K_{s}$, and $K_{f}$ are the bulk moduli of the drained frame, the solid phase, and the fluid phase, respectively.

\section{Spatial scales}

In this paper, we focus on fluid pressure diffusion at mesoscopic scales $l_{\text {meso }}$, which implies that the considered fracture lengths $l_{\text {frac }}$ are much larger than the pore size $l_{\text {pore }}$, but much smaller than the dominant seismic wavelength $\lambda$

$l_{\text {pore }} \ll l_{\text {frac }} \ll \lambda$.

The prevailing seismic wavelength defines the observation scale, generally referred to as the macroscopic scale, at which we want to assess the effective properties of the medium. Thus, the wavelength provides an upper limit for the area of investigation for obtaining meaningful effective properties through numerical volume averaging. The side length of the modelling domain $l_{\text {area }}$ therefore has to obey

$l_{\text {area }} \ll \lambda$.

Seismic waves propagating through a medium with mesoscopic heterogeneities induce fluid pressure diffusion at the interfaces of these heterogeneities. Biot's slow compressional wave is a proxy for this process and the characteristic scale of the mechanism is defined by the pressure diffusion length of the background medium:

$\lambda_{D}=\sqrt{\frac{\kappa}{\eta \omega} \frac{M L}{H}}$.

If $\lambda_{D}$ is much larger than the average distance between two consecutive fractures, there is enough time for pressure gradients to equilibrate, whereas if $\lambda_{D}$ is much smaller, there is no communication between fractures and the pore space of the background medium (Rubino et al. 2014). In these lowand high-frequency limits, the medium behaves as an elastic medium with a constant or piecewise constant fluid pressure. At all intermediate frequencies, velocity dispersion and attenuation occurs in response to pressure diffusion. Jänicke et al. (2015) argued that in this intermediate frequency regime a 
poroelastic medium can be replaced by an upscaled viscoelastic medium at the macroscopic scale if the diffusion length is smaller than the side length of the modelling domain, i.e.,

$\lambda_{D}<l_{\text {area }}$.

This implies that all diffusion processes take place in the mesoscopic scale range and no wave-induced fluid flow takes place at the macroscopic scale. Consequently, upscaling of Biot's quasi-static poroelastic equations involves spatial and temporal upscaling, leading to a homogeneous equivalent medium with time- and thus frequency-dependent effective properties.

Relations (6) and (8) provide constraints with regard to the side length of the modelling area considered in the upscaling procedure. Based on these relations, we can determine the effective frequency-dependent properties from the spatially averaged complex stress and strain fields by solving the coupled system of equations (1)-(4) under proper boundary conditions using a finite element approach (Rubino et al. 2009).

\section{D stiffness coefficients}

By applying a set of three oscillatory relaxation tests, comprising two compressional tests and one shear test, the equivalent frequency-dependent 2D stiffness coefficients are computed from the spatially averaged stress and strain fields

$$
\left(\begin{array}{c}
\left\langle\sigma_{11}(\omega)\right\rangle \\
\left\langle\sigma_{22}(\omega)\right\rangle \\
\left\langle\sigma_{12}(\omega)\right\rangle
\end{array}\right)=\left(\begin{array}{lll}
C_{11} & C_{12} & C_{13} \\
C_{12} & C_{22} & C_{23} \\
C_{13} & C_{23} & C_{33}
\end{array}\right)\left(\begin{array}{c}
\left\langle\epsilon_{11}(\omega)\right\rangle \\
\left\langle\epsilon_{22}(\omega)\right\rangle \\
\left\langle 2 \epsilon_{12}(\omega)\right\rangle
\end{array}\right) .
$$

The details of the procedure are described in the work of Rubino et al. (2015). Here, we slightly modify the boundary conditions. The two compressional tests are performed by imposing a time-harmonic homogeneous displacement on the upper boundary for test one and on the right lateral boundary for test two. Along the remaining boundaries, the sample is confined so that the solid phase is not allowed to have a normal displacement component relative to these boundaries. The third test is a pure shear test. In all three tests the sample is hydraulically sealed so that the fluid cannot flow in or out of the sample. A description of the used boundary conditions is given in Appendix. The resulting stiffness coefficients describe the effective behaviour of the entire heterogeneous medium provided that the considered sub-volume has at least the size of an REV.

\section{Representative elementary volume}

The classical definition of a representative elementary volume (REV) by Hill (1965) for elastic composites is that the inferred average properties of the medium are: (i) independent of the applied boundary conditions, and (ii) representative of the entire heterogeneous medium. More precisely, for an elastic medium a REV prevails if uniform displacement and uniform stress boundary conditions provide the same averaged moduli. Huet (1999) has shown that the same holds for viscoelastic media, and Jänicke et al. (2015) extended the concept to the upscaling of a heterogeneous poroelastic medium. Milani et al. (2016) studied boundary condition effects by performing creep (stress boundary condition) and relaxation (displacement boundary conditions) tests for sub-volumes of increasing size for a poroelastic medium with periodically distributed fractures. Here, we limit our investigation to displacement boundary conditions that provide an upper bound on the effective properties. Our aim is to analysze if these socalled apparent moduli (Ostoja-Starzewski 2006) represent the overall statistical properties of the underlying heterogeneous fractured medium.

To address this problem, we utilize the concept of the integral range for estimating the size of the REV as suggested by several authors (e.g. Willot and Jeulin, 2011; Kanit et al., 2003; Cailletaud et al., 1994). The integral range represents a domain over which the properties in a heterogeneous medium are correlated to each other. This in turn provides information on the domain size at which the average moduli are representative of the heterogeneous medium. This range can be estimated from the variogram or the covariance function of a random field and in a 2D medium has units of an area. For morphological parameters, such as the volume fraction or fracture density, the integral range can be calculated analytically for a given distribution. However, for elastic and viscoelastic moduli, it depends on the statistical properties of the stress and strain fields, which are not known a priori and can only be inferred from numerical simulations. To estimate the REV size of the frequency-dependent stiffness coefficients, we follow a combined statistical and numerical approach as proposed by Kanit et al. (2003) for elastic composite media.

We consider ensembles of finite-size random medium realizations for different domain sizes $S$. For each ensemble, we calculate the mean stiffness coefficients $\overline{C_{i j}}(S)$ and the corresponding variances from numerical simulations. The size of the modelling domain $S$ is large enough to represent an REV for the stiffness coefficients $C_{i j}$ if the variance of the average properties $C_{i j}(S)$ tends to zero, i.e., 
$\operatorname{Var}\left(C_{i j}(S)\right) \rightarrow 0$

Assuming ergodic and stationary stress and strain fields, the variance of the coefficients on the macroscopic scale can be expressed by the following scaling law (Cailletaud, Jeulin, and Rolland 1994)

$\operatorname{Var}\left(C_{i j}(S)\right)=\sigma^{2} \frac{A}{S}$,

where $\sigma^{2}$ is the point variance of $C_{i j}(S)$ at the mesoscopic scale, and $A$ is the integral range of the medium. The integral range can therefore be experimentally estimated from simulations of several realisations of the random medium for different domain sizes by fitting the scaling law (11) (e.g., Matheron 1971; Jeulin 1981). In the framework of this statistical approach, it is not possible to define a single minimum REV size. Instead, the size of the REV depends on the desired accuracy, as quantified by relative or absolute error, of the apparent moduli and the number of realizations of the random medium. The relative error of the mean apparent moduli $\overline{C_{i j}}(S)$ for a given number of realizations and a given size of the medium can be obtained from the confidence interval as

$\epsilon_{\text {rela }}=\frac{2 \sqrt{\operatorname{Var}\left(C_{i j}(S)\right)}}{\overline{C_{i j}}(S) \sqrt{n}}$,

where $n$ denotes the number of realizations. The corresponding absolute error is given by

$\epsilon_{a b s}=\frac{2 \sqrt{\operatorname{Var}\left(C_{i j}(S)\right)}}{\sqrt{n}}$.

Although the considered method does not yield a particular REV size, it still provides an indication whether an REV exists and how large the numerical cost is to obtain a certain accuracy with regard to the estimated moduli.

\section{UPSCALED STIFFNESS COEFFICIENTS}

We perform numerical simulations for three different domain sizes. The considered models comprise two different fracture densities and three different fracture lengths (Table 2). Earlier studies suggest that boundary condition effects are sensitive to the fracture density (Milani et al. 2016) as well as to the ratio between the edge length of the computational domain and the fracture length $\delta=l_{\text {area }} / l_{\text {frac }}$ (Saenger and Shapiro 2002). The number of model realizations $n$, the number of fractures per domain size $N / S$, the fracture volume fraction $V_{\text {frac }}$, the fracture density $\rho_{\text {frac }}$, and the scaling parameter $\delta$ are listed in Table 2 .

Figure 2 shows the mean value and variance of the stiffness coefficients over all model realizations (Table 2) obtained
Table 2 Number of numerical realisations for the four different fracture models and model characteristics

\begin{tabular}{llllll}
\hline Model & $\mathrm{N} / \mathrm{S}\left[\mathrm{m}^{2}\right]$ & $V_{\text {frac }}[\%]$ & $\rho_{\text {frac }}$ & $\delta$ & $n$ \\
\hline \multirow{2}{*}{ Base case } & $25 / 0.09$ & 0.8 & 0.16 & 6.25 & 40 \\
& $100 / 0.36$ & 0.8 & 0.16 & 12.5 & 20 \\
Dense case & $400 / 1.44$ & 0.8 & 0.16 & 25 & 5 \\
& $50 / 0.09$ & 1.6 & 0.32 & 6.25 & 40 \\
Length distribution & $200 / 0.36$ & 1.6 & 0.32 & 12.5 & 30 \\
case & $800 / 1.44$ & 1.6 & 0.32 & 25 & 5 \\
& $100 / 0.36$ & 0.8 & 0.208 & 3.125 & 60 \\
Orthogonal case & $500 / 1.44$ & 0.8 & 0.208 & 6.25 & 30 \\
& $50 / 0.09$ & 1.6 & 0.208 & 12.5 & 5 \\
& $200 / 0.36$ & 1.6 & 0.416 & 3.125 & 40 \\
& $800 / 1.44$ & 1.6 & 0.416 & 6.25 & 20 \\
& & & 12.5 & 5 \\
\hline
\end{tabular}

from the relaxation tests applied to domain sizes of $0.09 \mathrm{~m}^{2}$ and $1.44 \mathrm{~m}^{2}$. In all cases, the coefficients $C_{13}$ and $C_{23}$ are close to zero; thus, the symmetry of the fractured media is close to vertical transverse isotropy (VTI) for the horizontal fractures and hexagonal for the orthogonal fracture set. The coefficients $C_{22}$ and $C_{33}$ correspond to the P-wave modulus $\mathrm{H}^{v}$ for vertical compression along the top boundary and the shear wave modulus $G$, respectively. We observe that $C_{22}$ and $C_{33}$ decrease with an increase in the domain size, because the stress and strain fields at the boundaries are disturbed by applying a constant displacement. By increasing the domain size, these boundary effects are gradually averaged out. As expected, the boundary condition effects are larger for higher fracture densities and variable fracture lengths. In the latter case, the largest fracture length of $9.8 \mathrm{~cm}$ for the smallest domain size corresponds to a scaling parameter $\delta$ of 3.125 , which explains the larger boundary effects. This value of $\delta$ is significantly smaller than values reported in literature for which boundary effects become sufficiently small (Saenger and Shapiro 2002). Table 3 lists the absolute difference of the moduli between the smallest and largest domain size for $C_{22}$ and $C_{33}$. For the orthogonal case, additional boundary effects occur for $C_{11}$, the $\mathrm{P}$-wave modulus $\mathrm{H}^{b}$ for the horizontal compression and the coupling coefficient $C_{12}$ of the two P-wave moduli.

Boundary effects as well as the variance of the apparent $\mathrm{P}$-wave moduli $\mathrm{H}^{v}$ increase with decreasing frequencies as a result of fluid pressure diffusion between the fractures and the stiff pores of the background. At low frequencies, the fluid has time to flow out of the fracture into the background to equilibrate pressure differences; thus, the fracture 


\section{(a)}

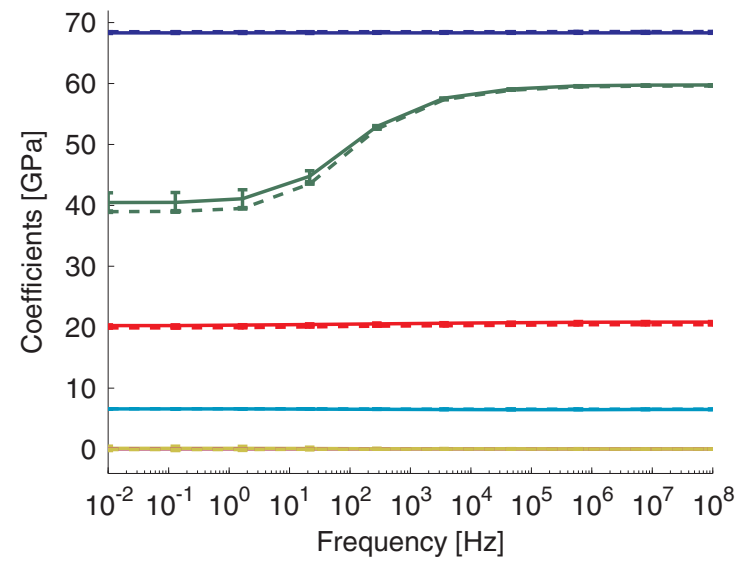

(c)

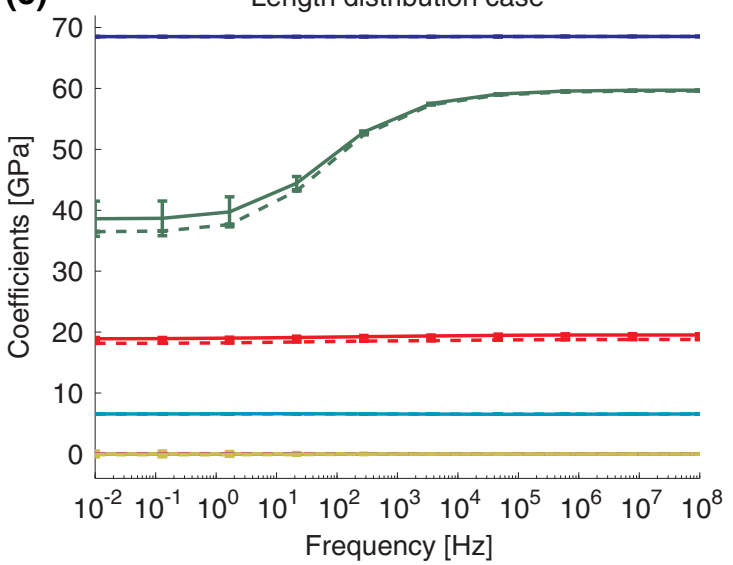

(b)

Dense case

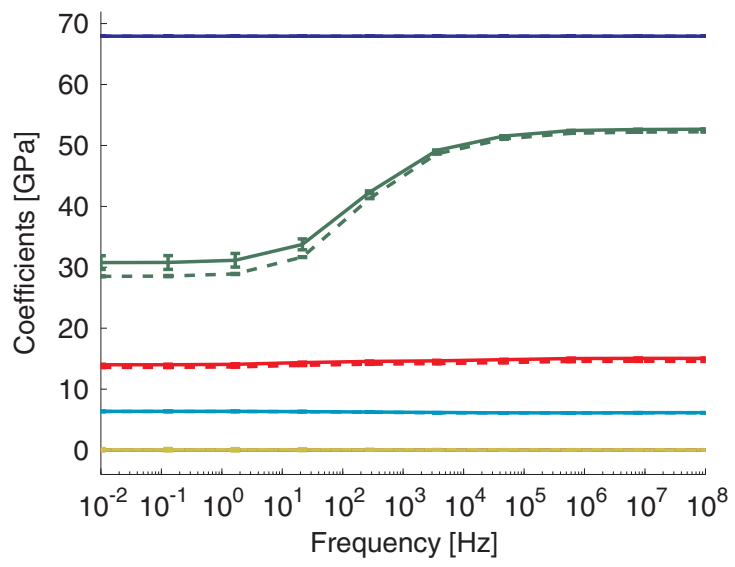

(d)

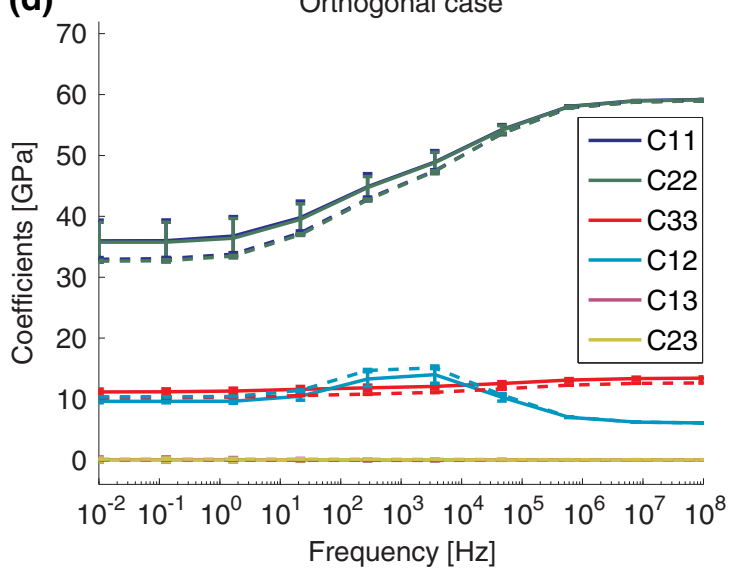

Figure 2 Mean value and variance of the real part of the stiffness coefficients for the domain sizes of $0.09 \mathrm{~m}^{2}$ (solid lines) and $1.44 \mathrm{~m}^{2}$ (dashed lines) over all model realisations listed in Table 2: base and dense cases with fracture densities of (a) $\rho_{\text {frac }}=0.16$ and (b) $\rho_{\text {frac }}=0.32$, respectively, and (c) length distribution and (d) orthogonal cases. The curves for the coefficients $\mathrm{C}_{13}$ and $\mathrm{C}_{23}$ are superimposed.

Table 3 Approximate absolute differences between the smallest and largest domain sizes for $C_{22}$ and $C_{33}$ at the lowest considered frequency

\begin{tabular}{lllll}
\hline & Base case & Dense case & $\begin{array}{l}\text { Length distribution } \\
\text { case }\end{array}$ & $\begin{array}{l}\text { Orthogonal } \\
\text { case }\end{array}$ \\
\hline$C_{22}($ small $)-C_{22}($ large $)$ & $1.5 \mathrm{GPa}$ & $2.13 \mathrm{GPa}$ & $2.25 \mathrm{GPa}$ & $3.12 \mathrm{GPa}$ \\
$\mathrm{C}_{33}($ small $)-C_{33}($ large $)$ & $0.4 \mathrm{GPa}$ & $0.75 \mathrm{GPa}$ & $0.48 \mathrm{GPa}$ & $1.03 \mathrm{GPa}$ \\
\hline
\end{tabular}

becomes more compliant. At high frequencies, no fluid exchange between the two regions occurs; hence, the fluid inside the fracture increases the resistance to compression. These stiffening effects of the fractures with increasing frequency reduce the compressibility contrast between the two regions and diminish boundary effects as well as the variance of the apparent P-wave moduli $\mathrm{H}^{v}$. The attenuation curves for the P-wave modulus $\mathrm{H}^{v}$ are shown in Fig. 3 for the largest domain size. The characteristic frequency of the pressure diffusion process corresponding to the peak attenuation depends on the diffusivity of the background medium (equation (7)) and the average distance between fractures. For the dense case, it is approximately $275 \mathrm{~Hz}$, and for all other cases, it is $20 \mathrm{~Hz}$. For the orthogonal case, an additional dispersion mechanism occurs between intersecting fractures at approximately $4.6 \mathrm{kHz}$ due to different pressure gradients in the vertical and horizontal fractures (Rubino et al. 2014; Quintal et al. 2014). 


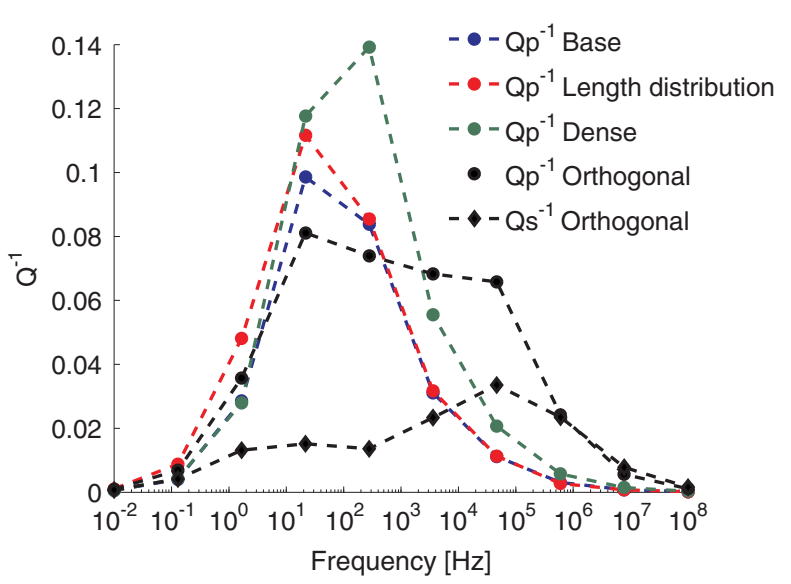

Figure 3 Attenuation of the P-wave modulus estimated for the mean value of the largest domain size for all four fracture distributions and for the $\mathrm{S}$-wave modulus of the orthogonal case.

For the S-wave modulus, we observe a small amount of dispersion due to the change of shape of the sample. This creates a dilation of the finite-sized fractures in the upper left and lower right corners and a compression of the fractures in the two opposite corners. Consequently, we observe in the former case a low pressure in the proximity of the fractures and in the latter case a high pressure. However, these small pressure differences lead to a negligible amount of attenuation, except for the case of orthogonal fractures where it might become noticeable (Fig. 3). As observed for the P-wave modulus an additional dispersion mechanism occurs at higher frequencies with a characteristic frequency of $4.6 \mathrm{kHz}$ due to the connectivity of fractures (Fig. 3). Since the S-wave modulus is not sensitive to the bulk modulus of the fluid, the contrast between the two regions and the variance of $\mathrm{C}_{33}$ and the boundary effects remain the same for all frequencies. A detailed description of boundary effects is given by Milani et al. (2016) and Jänicke et al. (2015), and an analysis of fractureto-fracture wave-induced fluid flow is given by Rubino et al. (2014) and Quintal et al. (2014). In the following, we focus on the statistical representativeness of the obtained apparent moduli.

\section{Estimation of the integral range}

For the four different fracture distributions (Table 2), we estimate the integral range by fitting the inferred variances of the moduli $\operatorname{Var}\left(H^{v}(S)\right)$ and $\operatorname{Var}(G(S))$, with the scaling law (11) for each frequency. This assumes that the real parts of the stress and strain fields are stationary at each frequency.
Figures 4 and 5 show the variance of the average moduli inferred from the simulations. As the scaling law (11) implies, the variance of the average moduli decreases with an increasing domain size, since the sample becomes statistically more representative. Furthermore, for all four cases, $\operatorname{Var}\left(H^{v}(S)\right)$ decreases with frequency, whereas $\operatorname{Var}(G(S))$ remains the same for the horizontal fractures and slightly decreases with frequency for orthogonal fractures. In order to utilize equation (11), we need the point variance of the P-wave and S-wave moduli. The point variance of the S-wave modulus (Kanit et al. 2003) is given by

$\sigma_{G}^{2}=\phi_{f r}\left(1-\phi_{f r}\right)\left(G_{f r}-G_{b}\right)^{2}$,

where $\phi_{f r}, G_{f r}$, and $G_{b}$ are the volume fraction of the fracture, the $\mathrm{S}$-wave modulus of the fracture (inverse of tangential compliance), and S-wave modulus of the background, respectively. The point variance of the $\mathrm{P}$-wave modulus varies with frequency since the normal compliance of the fracture is sensitive to the fluid pressure diffusion process and thus changes with frequency. Consequently, the frequency-dependent fracture compliance depends on the properties of the background medium and is difficult to calculate. However, the point variance in the high-frequency limit and for the dry case can be estimated as follows:

$\sigma_{H}^{2}=\phi_{f r}\left(1-\phi_{f r}\right)\left(H_{f r}-H_{b}\right)^{2}$,

$\sigma_{L}^{2}=\phi_{f r}\left(1-\phi_{f r}\right)\left(L_{f r}-L_{b}\right)^{2}$,

where $H$ is the saturated modulus of each phase, and $L$ is the dry modulus of each phase. The saturated modulus $H$ is obtained by applying Gassmann's equation (Gassmann 1951) to each phase separately. The two point variances $\sigma_{H}^{2}$ and $\sigma_{L}^{2}$ correspond to upper and lower limits, respectively. For simplicity, we utilized these two variances to estimate the integral range. This is justified since the difference between the two limits is small and the estimation of the integral range does not seem to be sensitive to the point variance (Fig. 6a).

The quality of the fitted scaling law (11) can be assessed based on Figs. 4 and 5, in which the simulated results and the fitted law are compared for the P- and S-wave moduli at all considered frequencies. Overall, the scaling law represents the simulations reasonably well. However, we observe that $\operatorname{Var}\left(H^{v}(S)\right)$ for the base case of the largest domain size is smaller than predicted by the scaling law (11). Furthermore, for the case with a length distribution, the simple scaling law seems to be no longer applicable since it is not able to capture 
(a)

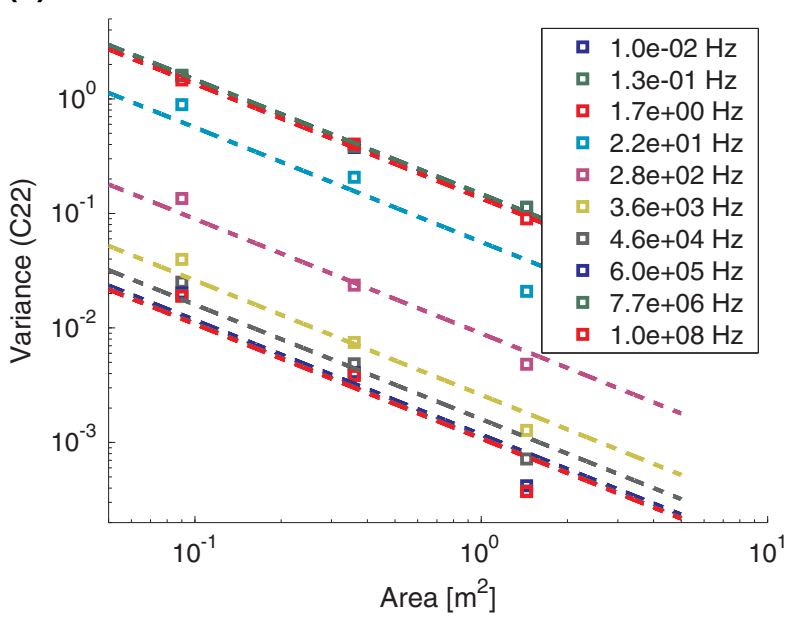

(c)

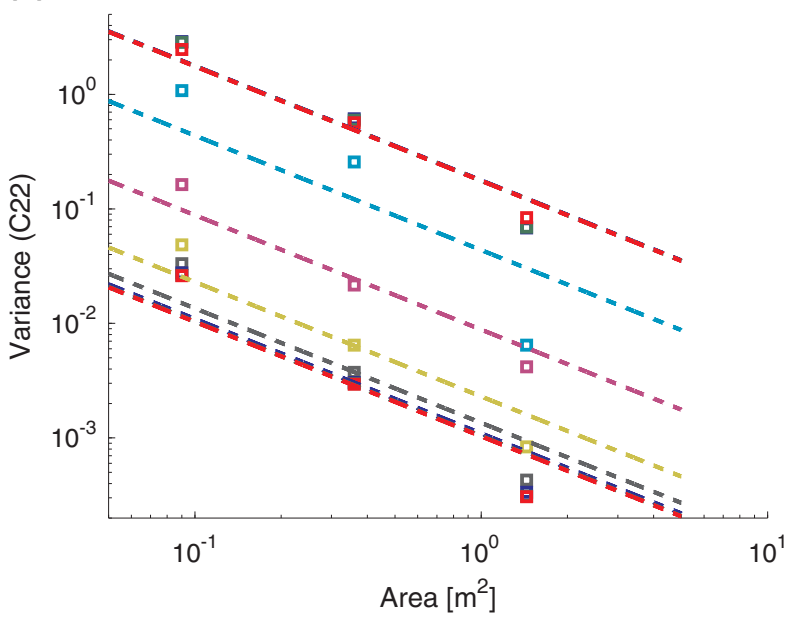

(b)

Dense case

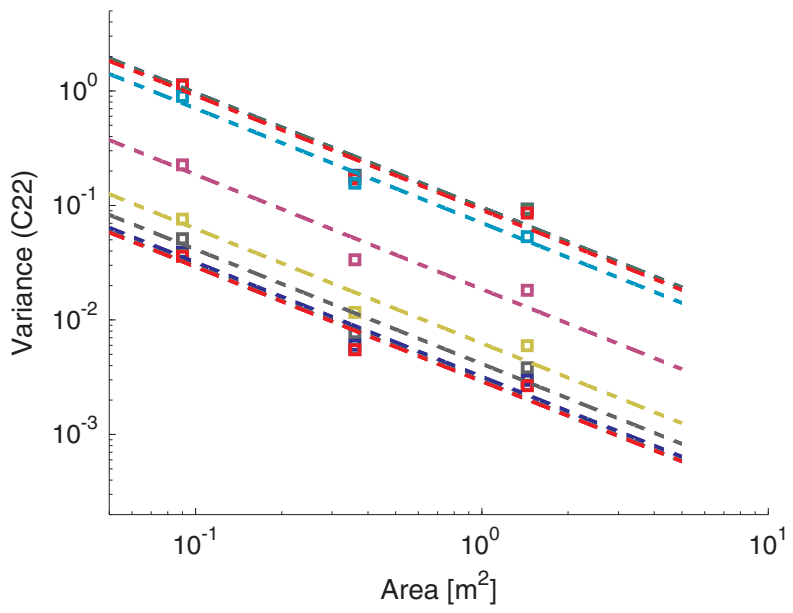

(d)

Orthogonal case

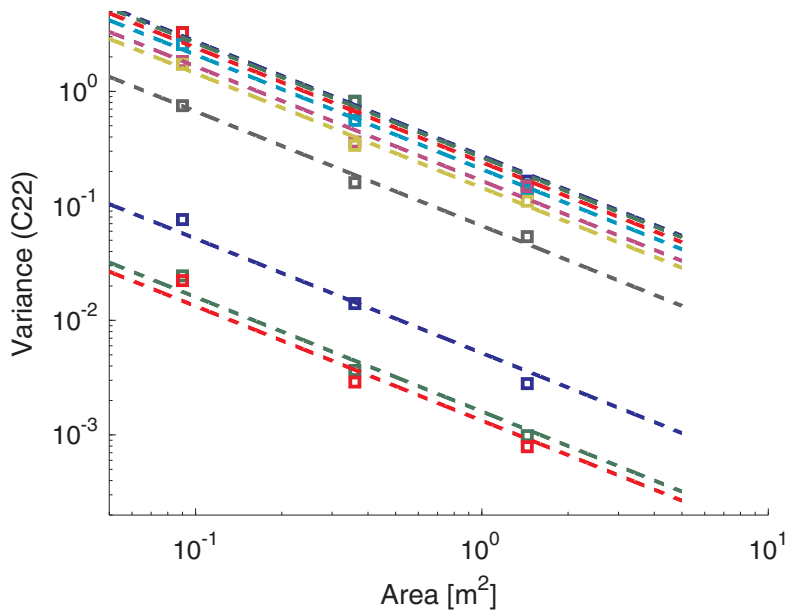

Figure 4 Comparisons of the estimated variance of the mean P-wave moduli for three medium sizes with the scaling law: (a) base case, (b) dense case, (c) length distribution and (d) orthogonal case. The number of realizations for each domain size is given in Table 2.

the change in the slope for higher frequencies. This might point to a scaling law of the form

$\operatorname{Var}\left(H^{v}(S)\right)=\sigma^{2}\left(\frac{A}{S}\right)^{\alpha}$

where $\alpha$ is an additional fitting parameter. A value of $\alpha$ larger than 1 implies a faster decrease of the variance, whereas a value smaller than 1 implies a slower decrease. Such a power law was indeed proposed by Kanit et al. (2003) and Cailletaud et al. (1994) with the justification that the change of scale is in general not additive. However, such an empirical scaling law does not allow for a straightforward interpretation of the integral range and hence is not further considered in this study.
In the following, we refer to $\sqrt{A}$ as the effective length to allow for a better comparison with the length scales of the fractured medium. The resulting effective lengths are plotted in Fig. 6. The estimates using $\sigma_{H}^{2}$ and $\sigma_{L}^{2}$ show only minor differences (Fig. 6a). Please note that the effective lengths for the P-wave modulus for the length distribution case are only reliable for the first two frequencies. Interestingly, at low frequencies, the effective lengths of the P-wave modulus for all four cases are on the order of the average vertical distances between horizontal fractures. This average distance is approximately $3 \mathrm{~cm}$ for the dense case and $6 \mathrm{~cm}$ for the other three cases. Furthermore, the decrease in the effective length with frequency is a mirror image of the dispersion curve. This makes intuitively sense since the integral range depends on 
(a)
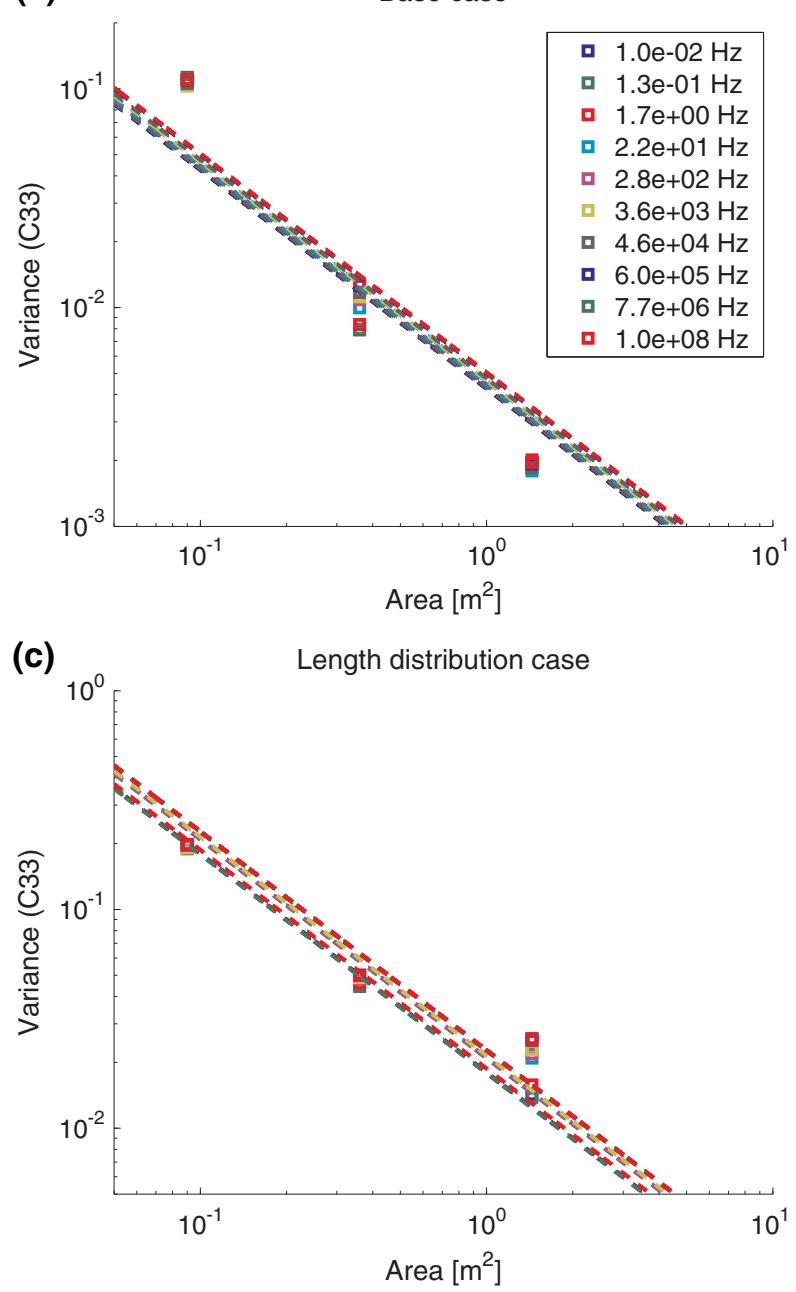

(b)

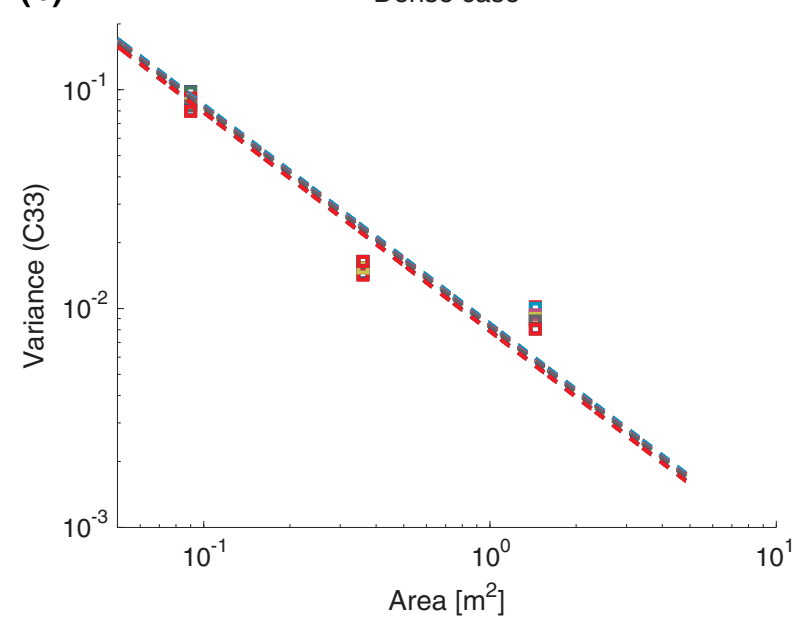

(d)

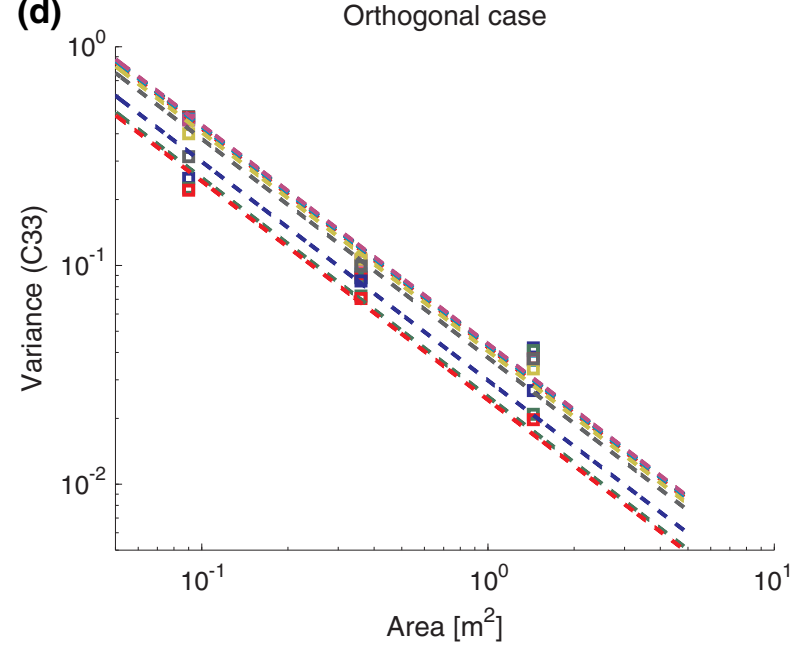

Figure 5 Comparisons of the estimated variance of the mean S-wave moduli for three domain sizes with the scaling law: (a) base case, (b) dense case, (c) length distribution, and (d) orthogonal case. The number of realization for each domain size is given in Table 2.

the volume fraction and on the elastic contrast between the fracture and background. This contrast decreases with frequency due to the stiffening of the fracture, and as a consequence, the interaction between fractures decreases. For the S-wave modulus, the effective lengths of the base and dense cases approximately correspond to the average distance between the fracture tips.

\section{Estimation of the REV size}

The fitted scaling law allows us to determine the size of the REV for a given relative or absolute error of the apparent moduli and a given number of realisations. Figures 7 and 8 show as a function of the sample area the number of realizations required to obtain a REV with a relative error of $1 \%$ (equation (12)) and an absolute error of $0.5 \mathrm{GPa}$ (equation (13)) of the apparent P-wave and S-wave moduli. The dashed grey lines indicate the size of the REV for five realizations. The estimations for the P-wave and S-wave moduli correspond to the lowest considered frequency. The estimated REV sizes indicate that five realizations of the largest considered domain $\left(1.44 \mathrm{~m}^{2}\right)$ are sufficient to obtain the apparent moduli with a relative error of less than $1 \%$ for the base, dense, and length distribution case. For the orthogonal case, a larger modelling domain or a larger number of realizations is required to obtain the same relative precision, especially in the case of the S-wave modulus. However, the REV sizes for an absolute error of $0.5 \mathrm{GPa}$ with the same number of simulations 

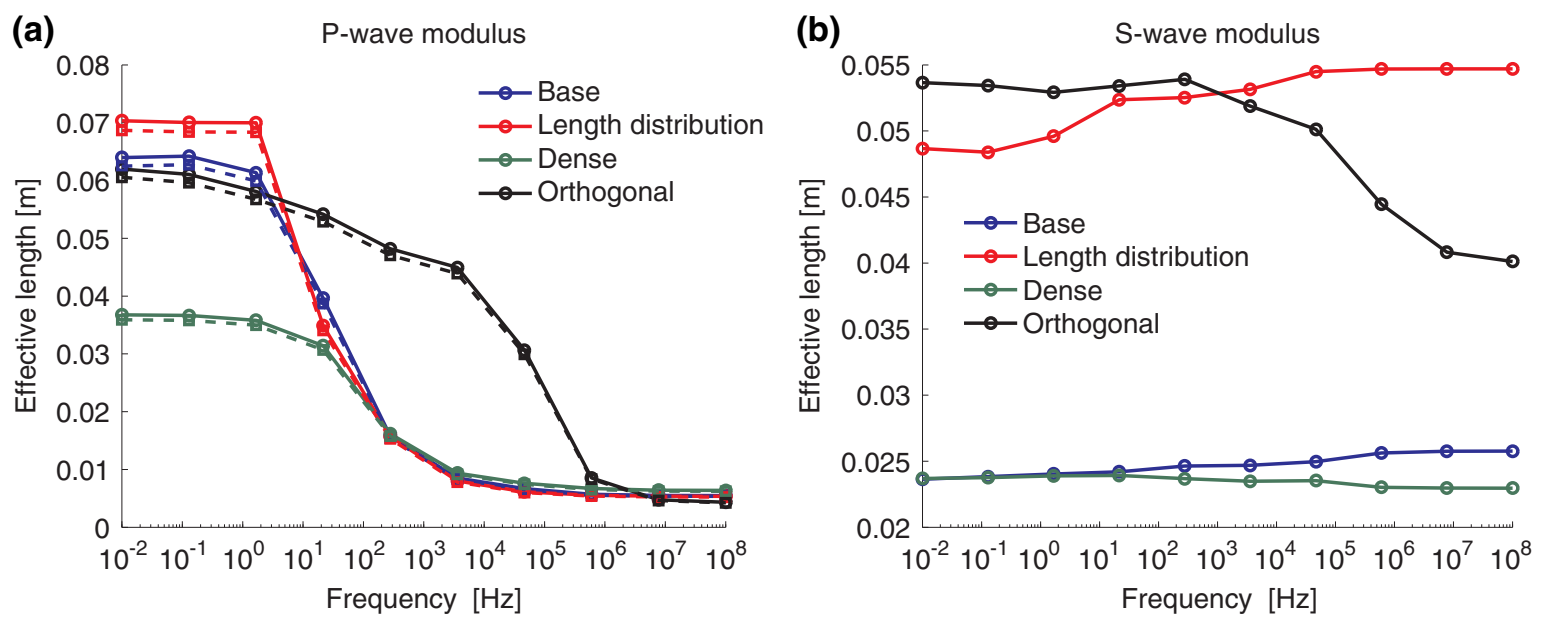

Figure 6 Estimated effective length for (a) the P-wave modulus and (b) the S-wave modulus as functions of frequency for all four fracture distributions. The dashed lines in (a) correspond to the estimations using $\sigma_{H}^{2}$ and the solid lines to those using $\sigma_{L}^{2}$.
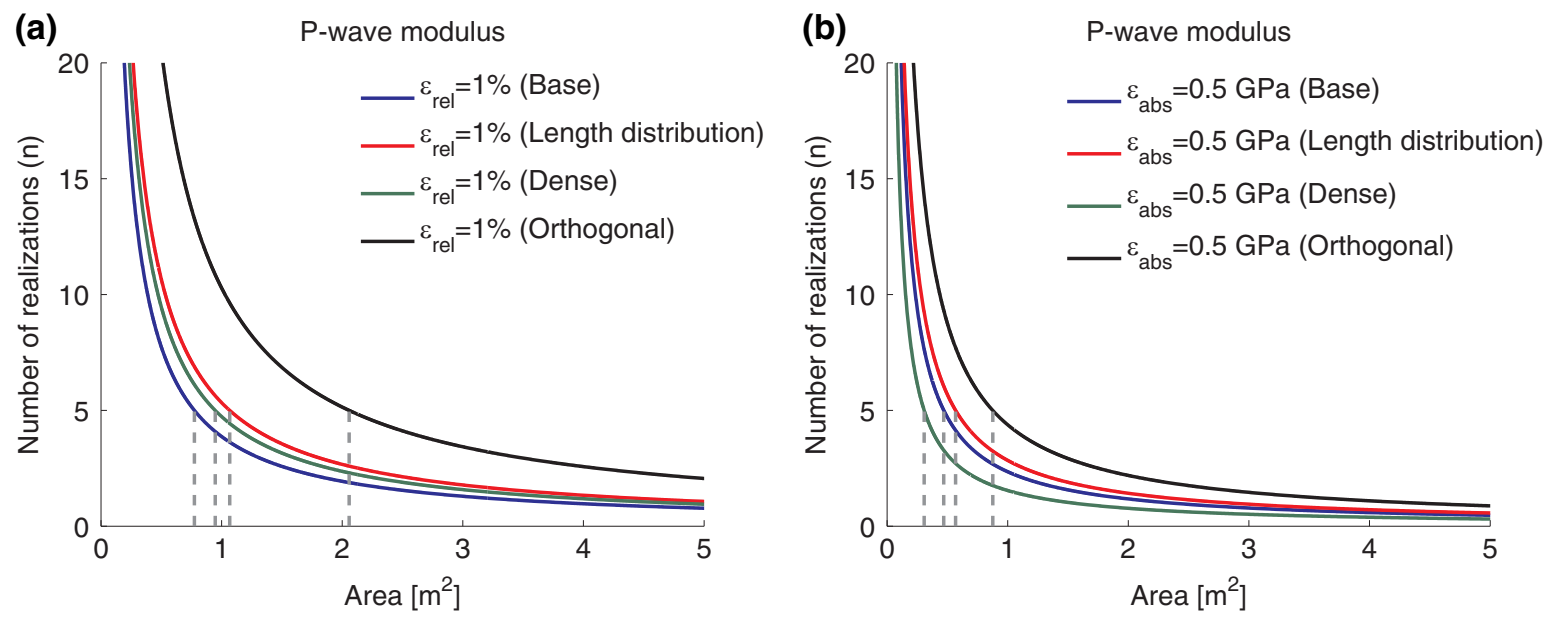

Figure 7 Number of realizations for (a) a relative error of $1 \%$ and (b) an absolute error of 0.5 GPa of the apparent P-wave modulus as a function of the domain size at the lowest frequency. The dashed grey lines indicate the size of the REV for five realizations.

are much smaller. In these context, it is, however, important to remember that, for these small modelling domains, strong boundary condition effects prevail, and the estimated apparent moduli provide only an upper bound of the true effective properties.

For the P-wave modulus, the REV size decreases with increasing frequency, as shown in Fig. 9 since the contrast between fractures and the host rock diminishes. Consequently, the variance of the apparent moduli is very small and, as discussed at the beginning of the section, the reduction in the compressibility contrast also diminishes the boundary effects. In fact, a difference of $0.5 \mathrm{GPa}$ is larger than the difference in apparent moduli between the smallest and largest domain sizes in the high-frequency limit. Hence, for an absolute error of $0.5 \mathrm{GPa}$, the REV size at high frequencies appears to be negligible.

\section{DISCUSSION}

Our results indicate that the overall statistical properties of the considered fracture distributions can be described with reasonable accuracy of the apparent moduli for computationally feasible REV sizes. We have limited the analysis to displacement boundary conditions, which only provide an upper bound on the effective properties. The decrease in the mean moduli with an increasing domain size does, however, 
(a)

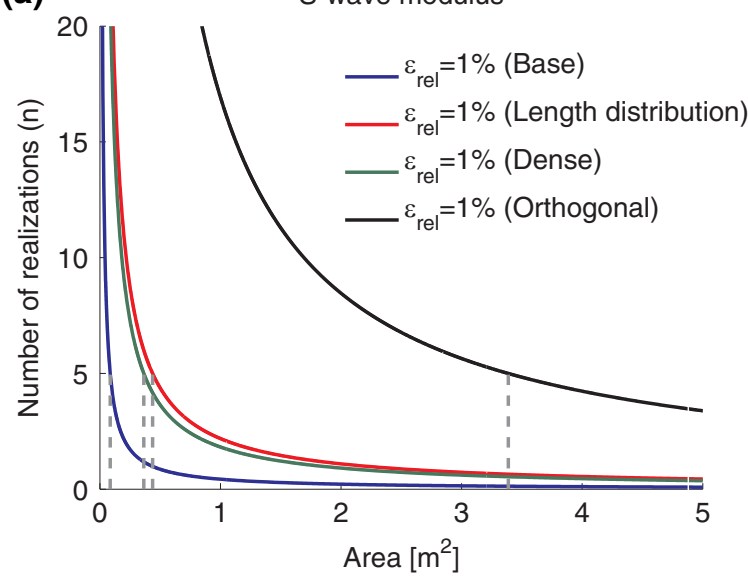

(b)

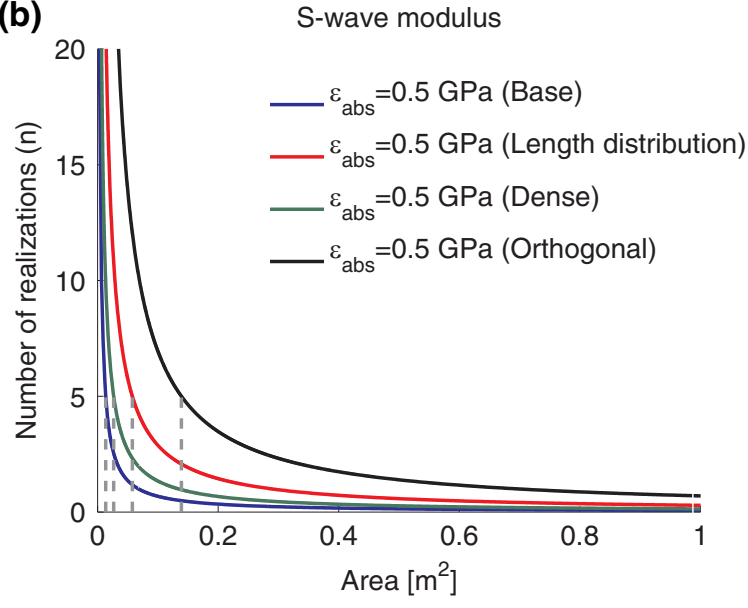

Figure 8 Number of realizations for (a) a relative error of $1 \%$ and (b) an absolute error of 0.5 GPa of the apparent S-wave modulus as function of the domain size at the lowest frequency. The dashed grey lines indicate the size of the REV for five realisations.

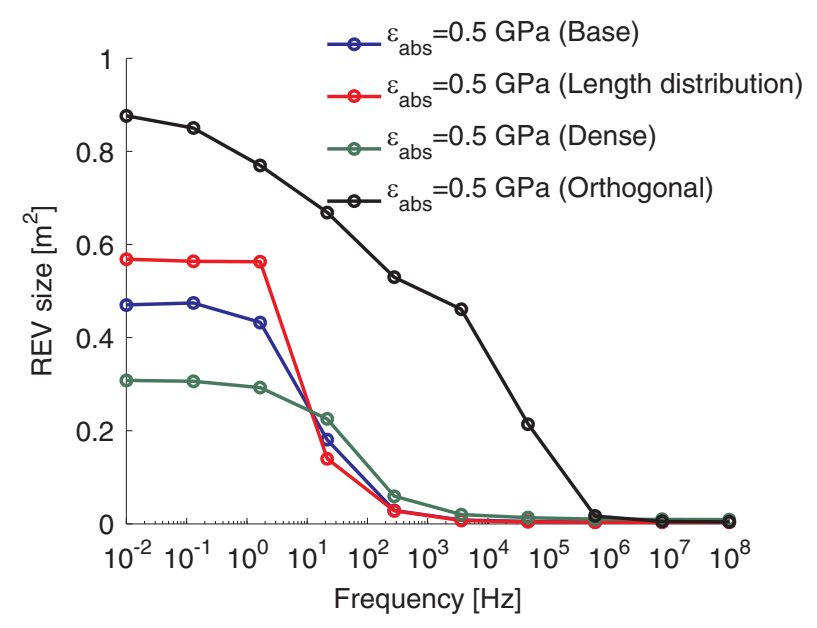

Figure 9 REV sizes for five realizations and an absolute error of $0.5 \mathrm{GPa}$ as function of frequency for the P-wave moduli.

provide indications with regard to the convergence rate to the effective properties (Fig. 10). It remains to be seen how large the REV size has to be to obtain effective properties of the medium that are independent of the applied boundary conditions. This will be investigated in a future study considering stress boundary conditions, which provide a lower bound on the effective properties.

Furthermore, for the length distribution case, the simple scaling seems to be no longer applicable. This might be due to the fact that changes of scale are not additive in general. Another reason might be that the domain sizes of $0.09 \mathrm{~m}^{2}$ and $0.36 \mathrm{~m}^{2}$ are too small for the largest fracture length of
$9.8 \mathrm{~cm}$, which corresponds to scaling parameters $\delta$ of 3.125 and 6.25, respectively. For comparison, Saenger and Shapiro (2002) have shown that in the case of pennyshaped cracks finite-size effects become sufficiently small for $\delta$ larger than 7 . Interestingly, the scaling law seems to hold for the orthogonal case with scaling parameters $\delta$ of 3.125 and 6.25 , although we observe the largest difference between the average moduli of the three domain sizes.

Another aspect which has to be taken into account to obtain meaningful effective properties by volume averaging are the restrictions on the modelling domain. In our study, the wavelengths of the $\mathrm{P}$ - and $\mathrm{S}$-waves become smaller than the largest domain size $l_{\text {area }}$ at frequencies of $\sim 400 \mathrm{~Hz}$ and $\sim 300 \mathrm{~Hz}$, respectively, and thus violate the upper limit $l_{\text {area }} \ll \lambda$. At higher frequencies, a wave travelling through such a medium scans the entire medium, but it is only sensitive to a certain part of the medium at any given point in time. Conversely, the response of the stress and strain fields to an oscillatory displacement takes place everywhere in the medium at once but is confined to the immediate vicinity of the heterogeneities. If the stress and strain fields are ergodic, i.e., if ensemble averaging (passage of the wave) and volume averaging (oscillatory test) are interchangeable, both approaches should lead to the same effective properties as long as the considered frequencies are below Biot's critical frequency and no scattering effects occur.

The combined statistical and numerical approach allows us to estimate REV sizes of the frequency-dependent stiffness coefficients. In general, the REV size depends on the physical 
(a)

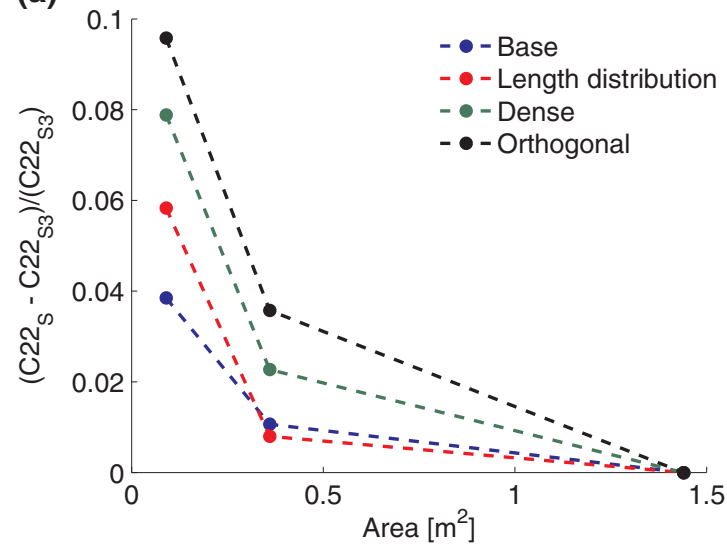

(b)

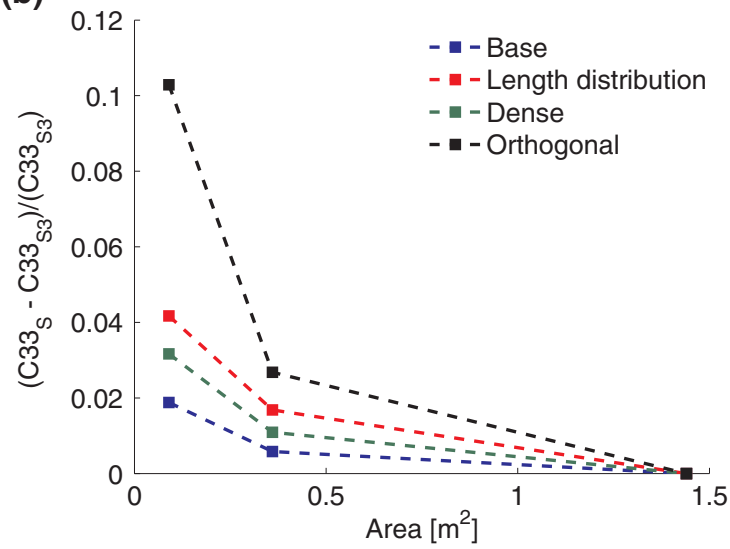

Figure 10 Relative difference of (a) the P-wave modulus and (b) the S-wave modulus with respect to the largest domain size (S3) as functions of the domain size.

property as shown for the P- and S-wave moduli. Hence, the REV size for the attenuation might differ from the estimated sizes for the stiffness coefficients. However, an extension of the analysis for attenuation is not straightforward since one would need to estimate its point variance that cannot be obtained analytically. The problem already arose for the P-wave modulus that can only be represented by a two-phase medium in the dry case and high-frequency limit. Both of these approximations provide very similar estimates of the REV size (Fig. 6), which does however not guarantee that we adequately capture the variability of the medium at the mesoscopic scale. The frequency-dependent fracture compliance depends on the state of the fluid pressure in the entire medium and thus on the properties of the background medium. A more suitable approximation of the point variance and its changes with frequency might thus be obtained from the simulated stress fields. This would open an avenue towards studying the variability of the real and imaginary parts of the stress field, which in turn are linked to the dispersion and attenuation of the coefficients. Furthermore, it has to be clarified if the assumption of stationarity holds for the complex stress fields.

In this study, we considered 2D numerical models under plane strain conditions. This is equivalent to corresponding $3 \mathrm{D}$ media with infinite fracture planes in the third dimension, i.e., outside the 2D modelling plane. Furthermore, we assumed a uniform distribution of fracture centres for parallel and orthogonal fracture sets. In spite of their simplicity, these fracture networks still provide insights into the upscaling problem of water-saturated fractured media, and this represents a first step towards the estimation of effective frequency-dependent moduli of more realistic fracture networks.

\section{ACKNOWLEDGEMENTS}

This work has been completed within the Swiss Competence Center on Energy Research - Supply of Electricity, with the support of the Swiss Commission for Technology and Innovation. T.M Müller greatly acknowledges the financial support of the Foundation Herbette for his visit at the University of Lausanne.

\section{REFERENCES}

Biot M. 1941. General theory of three-dimensional consolidation. Journal of Applied Physics 12, 155-164.

Brajanovski M., Müller T.M. and Gurevich B. 2006. Characteristic frequencies of seismic attenuation due to wave-induced fluid flow in fractured porous media. Geophysical Journal International 166, 574-578.

Cailletaud G., Jeulin D. and Rolland Ph. 1994. Size effect on elastic properties of random composites. Engineering Computations 11, 99-110.

Chapman M. 2003. Frequency-dependent anisotropy due to mesoscale fractures in the presence of equant porosity. Geophysical Prospecting 51, 369-379.

Gassmann F. 1951. Über die Elastizität poröser Medien. Vierteljahrsschrift der Naturforschenden Gesellschaft in Zürich 96, 1-23.

Gurevich B., Brajanovski M., Galvin R., Müller T.M. and TomsStewart J. 2009. P-wave dispersion and attenuation in fractured and porous reservoirs -poroelasticity approach. Geophysical Prospecting 57, 225-237.

Hill R. 1965. A self-consistent mechanics of composite materials. Journal of the Mechanics and Physics of Solids 13, 213-222.

Huet C. 1999. Coupled size and boundary-condition effects in viscoelastic heterogeneous and composite bodies. Mechanics of Materials 31, 787-829.

Ishimaru A. 1978. Wave Propagation and Scattering in Random Media. Academic Press. 
Jaeger J.C., Cook N.G.W. and Zimmerman R.W. 2007. Fundamentals of Rock Mechanics. Blackwell Publishing.

Jänicke R., Quintal B. and Steeb H. 2015. Numerical homogenization of mesoscopic loss in poroelastic media. European Journal of Mechanics -A/Solids 49, 382-395.

Jeulin D. 1981. Mathematical morphology and multiphase materials. In: Proceedings of the 3rd European Symposium for Stereology, Ljubljana, Yugoslavia, June 22-27, 1981. pp. 265-286.

Kachanov M. 1992. Effective elastic properties of cracked solids: critical review of some basic concepts. Applied Mechanics Reviews 45, 304-335.

Kanit T., Forest S., Galliet I., Mounoury V. and Jeulin D. 2003. Determination of the size of the representative volume element for random composites: statistical and numerical approach. International Journal of Solids and Structures 40, 3647-3679.

Matheron G. 1971. The Theory of Regionalized Variables and Its Applications, pp. 5. École National Supérieure des Mines.

Mavko G., Mukerji T. and Dvorkin J. 2009. The Rock Physics Handbook: Tools for Seismic Analysis of Porous Media. Cambridge University Press.

Milani M., Rubino J.G., Müller T.M., Quintal B., Caspari E. and Holliger K. 2016. Representative elementary volumes for evaluating effective seismic properties of heterogeneous poroelastic media. Geophysics 81(2), D169-D181.

Ostoja-Starzewski M. 1999. Scale effects in materials with random distributions of needles and cracks. Mechanics of Materials 31, 883-893.

Ostoja-Starzewski M. 2006. Material spatial randomness: from statistical to representative volume element. Probabilistic Engineering Mechanics 21, 112-132.

Quintal B., Jänicke R., Rubino J.G., Steeb H. and Holliger K. 2014. Sensitivity of S-wave attenuation to the connectivity of fractures in fluid-saturated rocks. Geophysics 79, WB15-WB24.

Quintal B., Steeb H., Frehner M., and Schmalholz S.M. 2011. Quasistatic finite element modeling of seismic attenuation and dispersion due to wave-induced fluid flow in poroelastic media. Journal of Geophysical Research 116(B01).

Rubino J.G., Caspari E., Milani M., Müller T.M. and Holliger K. 2015. Seismic anisotropy in fractured low-permeability formations: The effects of hydraulic connectivity. 85th SEG meeting, New Orleans, USA, Expanded Abstracts, 3219-3223.

Rubino J.G., Guarracino L., Müller T.M. and Holliger K. 2013. Do seismic waves sense fracture connectivity? Geophysical Research Letters 40, 692-696.

Rubino J.G., Müller T.M., Guarracino L., Milani M. and Holliger K. 2014. Seismoacoustic signatures of fracture connectivity. Journal of Geophysical Research 119, 2252-2271.

Rubino J.G., Ravazzoli C.L. and Santos J.E. 2009. Equivalent viscoelastic solids for heterogeneous fluid-saturated porous rocks. Geophysics 74, N1-N13.

Saenger E.H., Krüger O.S. and Shapiro S.A. 2006. Effective elastic properties of fractured rocks: dynamic vs. static considerations. International Journal of Fracture 139, 569-576.

Saenger E.H. and Shapiro S.A. 2002. Effective velocities in fractured media: a numerical study using the rotated staggered finitedifference grid. Geophysical Prospecting 50, 183-194.
Sanchez-Vila X., Guadagnini A. and Carrera J. 2006. Representative hydraulic conductivities in saturated groundwater flow. Reviews of Geophysics 44.

Schoenberg M. and Sayers C.M. 1995. Seismic anisotropy of fractured rock. Geophysics 60, 204-211.

Wenzlau F., Altmann J. and Müller T.M. 2010. Anisotropic dispersion and attenuation due to wave-induced fluid flow: quasi-static finite-element modeling in poroelastic solids. Journal of Geophysical Research 115(B07).

Willot F. and Jeulin D. 2011. Elastic and electrical behavior of some random multiscale highly contrasted composites. International Journal for Multiscale Computational Engineering 9.

\section{APPENDIX}

\section{BOUNDARY CONDITIONS}

To estimate the frequency-dependent stiffness coefficients, we solve Biot's 1941 quasi-static poroelastic equations (1)-(4) using the following displacement boundary conditions. The computational domain is given by $\Omega=\left[0, L_{x}\right] \times\left[0, L_{z}\right]$ with the boundary $\Gamma=\Gamma^{L} \cup \Gamma^{B} \cup \Gamma^{R} \cup \Gamma^{T}$, where

$\Gamma^{L}=\{(x, z) \in \Omega: x=0\}$,

$\Gamma^{R}=\left\{(x, z) \in \Omega: x=L_{x}\right\}$,

$\Gamma^{B}=\{(x, z) \in \Omega: z=0\}$,

$\Gamma^{T}=\left\{(x, z) \in \Omega: z=L_{z}\right\}$.

The boundary conditions for the two compressional tests are given by

$$
\begin{aligned}
& \boldsymbol{u} \cdot \boldsymbol{v}=-\Delta u,(x, z) \in \Gamma^{T}, \\
& \left.\boldsymbol{u} \cdot \boldsymbol{v}=0,(x, z) \in \Gamma^{L} \cup \Gamma^{R} \cup \Gamma^{B} \quad \text { (Test } 1\right), \\
& \boldsymbol{u} \cdot \boldsymbol{v}=-\Delta u,(x, z) \in \Gamma^{R}, \\
& \left.\boldsymbol{u} \cdot \boldsymbol{v}=0,(x, z) \in \Gamma^{L} \cup \Gamma^{T} \cup \Gamma^{B} \quad \text { (Test } 2\right), \\
& \boldsymbol{w} \cdot \boldsymbol{v}=0,(x, z) \in \Gamma,
\end{aligned}
$$

with the compatible condition

$(\sigma \boldsymbol{v}) \cdot \chi=0,(x, z) \in \Gamma$,

where $v$ and $\chi$ denote the unit outer normal and unit tangent on $\Gamma$ so that $\{\boldsymbol{v}, \boldsymbol{\chi}\}$ is an orthonormal system on $\Gamma$. The third test is a pure shear test with the following boundary conditions

$$
\begin{aligned}
& u \cdot \boldsymbol{\chi}=\Delta u,(x, z) \in \Gamma^{T} \cup \Gamma^{B}, \\
& \boldsymbol{u} \cdot \boldsymbol{v}=\Delta u \cdot x,(x, z) \in \Gamma^{T} \cup \Gamma^{B}, \\
& \boldsymbol{u} \cdot \boldsymbol{v}=\Delta u \cdot z,(x, z) \in \Gamma^{L} \cup \Gamma^{R}, \\
& \boldsymbol{u} \cdot \boldsymbol{\chi}=\Delta u,(x, z) \in \Gamma^{L} \cup \Gamma^{R}, \\
& \boldsymbol{w} \cdot \boldsymbol{v}=0,(x, z) \in \Gamma,
\end{aligned}
$$

